\title{
The LROSE Science Gateway: One-Stop Shop for Weather Data, Analysis, and Expert Advice
}

\author{
Brenda Javornik \\ National Center for Atmospheric \\ Research \\ Boulder, Colorado, USA \\ brenda@ucar.edu
}

\author{
Hector Santiago III \\ Winston-Salem State University \\ Clemmons, North Carolina, USA \\ hector.santiagomarti@gmail.com
}

\author{
Jennifer C. DeHart \\ Colorado State University \\ Fort Collins, Colorado, USA
}

\begin{abstract}
Nexrad data along with software to convert between binary formats, perform quality control, analyze, and visualize the data are all public and open for access and download. What is missing is the knowledge of how to use the available components with reproducible results. A science gateway provides a web-based platform to bring all the components together. Where a novice can learn from experts, and where expert researchers can customize software tools for atmospheric science.
\end{abstract}

\section{CCS CONCEPTS}

- Applied computing $\rightarrow$ Earth and atmospheric sciences; • Computing methodologies $\rightarrow$ Distributed computing methodologies.

\section{KEYWORDS}

Nexrad, radar, lidar, atmospheric science, cloud computing, softwareas-a-service, Apache Airavata, science gateways

\section{ACM Reference Format:}

Brenda Javornik, Hector Santiago III, and Jennifer C. DeHart. 2021. The LROSE Science Gateway: One-Stop Shop for Weather Data, Analysis, and Expert Advice. In Practice and Experience in Advanced Research Computing (PEARC '21), fuly 18-22, 2021, Boston, MA, USA. ACM, New York, NY, USA, 6 pages. https://doi.org/10.1145/3437359.3465595

\section{INTRODUCTION}

The entire National Weather Service Next-Generation Radar (Nexrad) data set is now available from Amazon Web Services, Google Cloud, and Open Commons Consortium [19]. The Nexrad system comprises 160 sites throughout the world. Each site produces "the base meteorological data quantities of reflectivity, mean radial velocity, and spectrum width, as well as the dual-polarization base data of differential reflectivity, correlation coefficient, and differential phase" [17]. Each site produces data files at four, five, six, or ten minute intervals. One file is approximately six megabytes. Each file contains 360-degree sweeps of the antenna at multiple elevation angles [18]. The Nexrad Level II data are noisy and need cleaning

Permission to make digital or hard copies of all or part of this work for personal or classroom use is granted without fee provided that copies are not made or distributed for profit or commercial advantage and that copies bear this notice and the full citation on the first page. Copyrights for components of this work owned by others than ACM must be honored. Abstracting with credit is permitted. To copy otherwise, or republish, to post on servers or to redistribute to lists, requires prior specific permission and/or a fee. Request permissions from permissions@acm.org.

PEARC '21, fuly 18-22, 2021, Boston, MA, USA

(C) 2021 Association for Computing Machinery.

ACM ISBN 978-1-4503-8292-2/21/07 ..\$15.00

https://doi.org/10.1145/3437359.3465595 and quality control before analysis. The software tools require expert knowledge to marshal the data through the various stages of quality control and analysis. Radar data are time-series data, which means the data are indexed and referenced by date and time. The raw, binary data become too detailed and numerous to detect trends without visualization.

The Lidar Radar Open Source Environment (LROSE) [3] aims to develop and maintain high-quality $\mathrm{C}++$ based applications for quality control, visualization, and analysis by the radar and lidar research community. LROSE provides command-line and graphical interface tools. The tools fall into four main categories: (a) data format translation, to convert most radar and lidar binary data formats into a standard NetCDF-based CfRadial format [7]; (b) coordinate transformations, to interpolate observations in radar polar coordinates into Cartesian space; (c) algorithms for quality control and to create scientific products; and (d) displays for data visualization. In addition, LROSE has applications for Doppler wind retrieval and analysis, from both single and multiple radar observations.

LROSE is part of the Open Radar Software $[9,11]$ effort that supports geoscience applications for the weather radar community. There are 78 unique institutions registered for the software, including domestic and international. The LROSE community identify themselves as researchers (at $55 \%$ ), $10 \%$ as educators, $27 \%$ as students, $3 \%$ as developers, and $5 \%$ as something else, such as commercial companies. The community comprises mainly atmospheric science researchers and students.

The problem is how to make data, analysis software, and expert knowledge available to the atmospheric science research and education community, while also making the access easier, with no software installation issues and no unique hardware and software environments that may not be reproducible. The problem is common across many areas of science, however, the expert knowledge is unique to the atmospheric sciences. An open virtual machine for cross-platform weather radar science [10] works to overcome the barriers of installing software on various operating systems. Amazon Web Services Public Sector references WeatherPipe [2, 8] to display Nexrad data.

A science gateway can help. "Science gateways allow science and engineering communities to access shared data, software, computing services, instruments, educational materials, and other resources specific to their disciplines" [14]. Within a gateway, tutorials as workflows could embed the expertise needed. A workflow is a guide to the order of the appropriate tools, with example data, expected output, parameter settings, and access to relevant data sources. The gateway provides the mechanism and platform for an interactive learning experience. 
A science gateway solution brings together the LROSE tool set with the assistance from Science Gateways Community Institute to develop a prototype gateway environment for weather radar analysis and visualization. The LROSE science gateway would make radar analysis in the cloud possible. A science gateway offers the advantage of access to useful tools along with relevant data in one place without the need to upload and download large amounts of data. A gateway provides the framework for reproducible science and data analysis. There is no installation of software on local machines. A researcher can access Nexrad data directly through the gateway and perform analyses entirely in the cloud. A gateway also offers a teaching and learning environment where established scientific workflows are accessible to students and new faculty. The university staff could set up realistic exercises and tutorials for their students.

The ideal audience for a science gateway includes students for the tutorial workflows and researchers for the individual tools. Educators could make use of both workflows and individual tools by creating new tutorials. Commercial users and developers could create applications that access the gateway directly as a web service, which makes the gateway a service ( "Gateway, application as a service.")

Scientists at the National Center for Atmospheric Research (NCAR) and Colorado State University (CSU) developed tutorials common for weather radar analysis. The tutorials provide a guide for rainfall estimation and two types of wind analysis. The initial LROSE Science Gateway would present the LROSE tools as individual applications, and the tutorials as interactive workflows. Figure 1 is the LROSE Gateway landing page. The three workflows feature prominently in the page with a brief description. A discretely placed link toward the bottom of the page provides access to the individual tools.

\section{LROSE SCIENCE GATEWAY}

The LROSE Science Gateway development follows the usual phases of initial design based on the anticipated user experience, implementation, integration, and deployment of a prototype gateway.

\subsection{Design}

A six week consultation $[15,22]$ with the SGCI user experience and user interface (UX/UI) team revealed the need for separate access points for the main categories of users. Experienced atmospheric researchers would directly access the individual applications. Whereas, students and novice researchers could use workflows to learn the sequence of tools for analysis.

The UX/UI group designed an example access point for the individual tools as shown in Figure 3. The individual LROSE tools run as standalone applications in the gateway. Each application execution becomes a separate experiment with input data, output data and a parameter file. The LROSE software suite contains over 40 individual tools.

The UX/UI team also designed a detailed interface for the workflows. Figure 2 shows the third stage of the Wind Analysis workflow. The workflow design is a general template and applies to all the workflows. The template has multiple stages with data visualization at each stage.

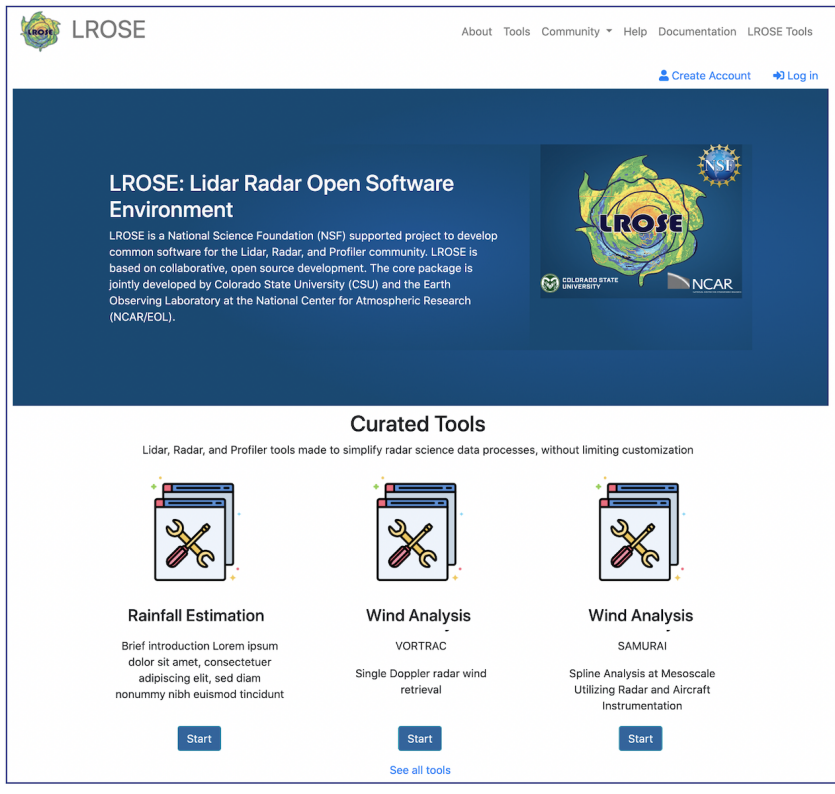

Figure 1: The LROSE Science Gateway. Three curated tools present the workflow tutorials. The individual LROSE tools are not as obvious, but are still available from the See all tools link.

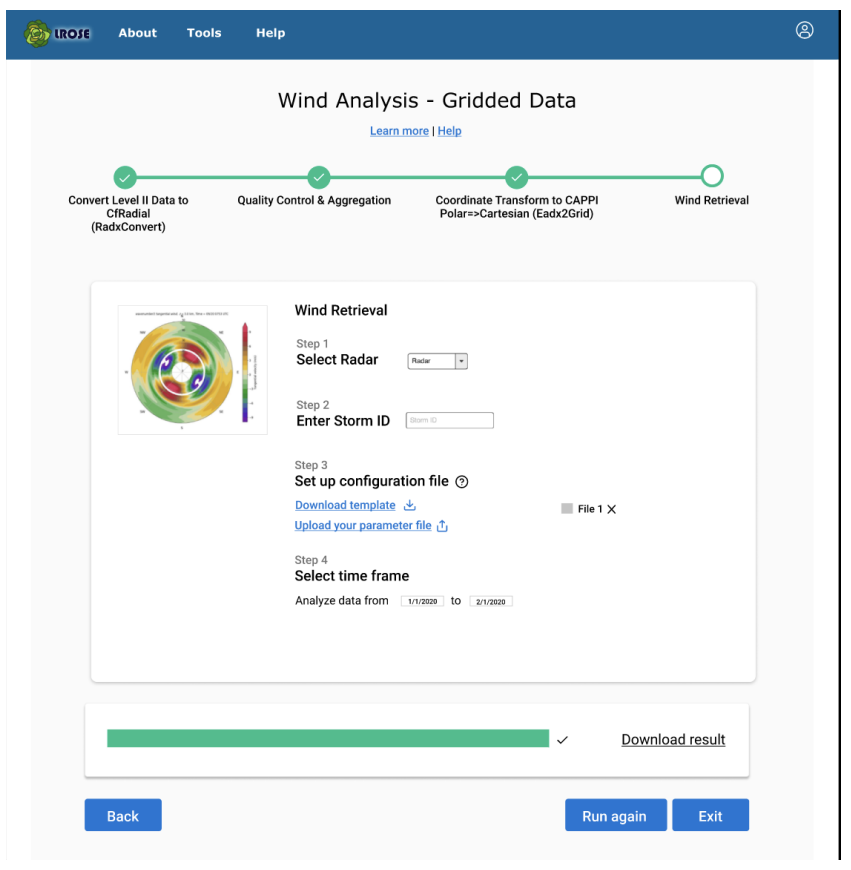

Figure 2: The Wind Analysis Workflow designed by UX/UI team.

The remainder of this section describes the development of the surface precipitation estimation workflow. The precipitation workflow has three major stages: data format conversion (RadxConvert), 


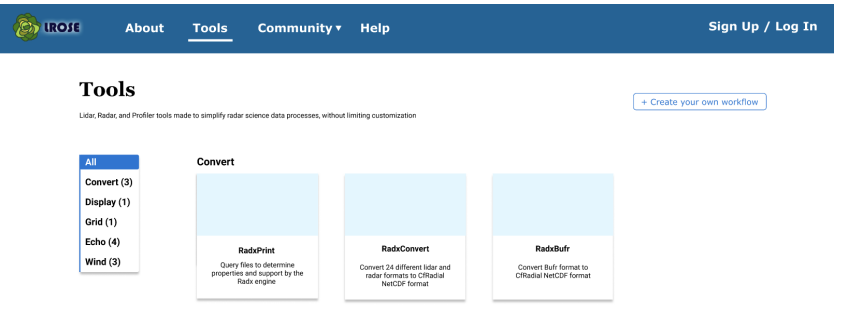

Figure 3: LROSE individual tools designed by UX/UI team.

processing and three-dimensional precipitation rate estimation (RadxRate), and final quality control and two-dimensional surface precipitation rate estimation (RadxQpe). The goal is an interface that is simple and obvious to use. In practice, additional, optional steps calculate beam blockage, and determine rain rate accumulation. The first implementation of the workflow omits these optional steps and provides the users with a beam blockage file.

Each stage of the precipitation workflow uses at least one LROSE processing tool and one LROSE visualization tool. The LROSE tools RadxPrint, HawkEye, and Cartesian Interactive Data Display (CIDD) output text and images for visual inspection of the binary data. Parameter file settings customize each LROSE tool.

Stage one of the precipitation workflow converts raw data files into a common format for the other LROSE applications. This stage requires a start time, stop time, the name of a Nexrad radar station, and a parameter file for the LROSE tool RadxConvert. From this information, an application pulls the requested Nexrad data files from AWS servers into the gateway. RadxConvert reformats the raw data files into the CfRadial format that the other LROSE tools can use [7]. The LROSE visualization tool, HawkEye, ingests CfRadial data and generates images for the most common data fields: reflectivity, velocity, etc.

Stage two of the precipitation workflow processes the converted file to estimate the precipitation rate for each gate within the threedimensional radar volume. This stage requires the CfRadial data files from stage one and specific parameter files as input to the LROSE tool RadxRate. The results are binary data files in CfRadial format. HawkEye reads the binary files, and generates images for inspection.

Stage three of the workflow estimates the final precipitation rate closest to the ground. This stage requires the three-dimensional results of stage two, beam blockage data, a parameter file specific for the RadxQpe tool and produces output data files in polar and Cartesian grids. HawkEye produces images for the polar data. CIDD produces images for the Cartesian data. The visualization tools HawkEye and CIDD read the CfRadial binary data formats and produce standard image files, such as PNG, JPG, and TIFF.

At each stage if the results do not appear satisfactory, a novice could modify the parameter file and repeat the stage until the results are acceptable. The workflow then continues with the automatic flow of data into the next stage.

\subsection{Implementation}

Through SGCI, we were directed to SciGaP [20] gateway platform. $\mathrm{SciGaP}$ is a hosted gateway platform with a user facing Django portal [5] and Apache Airavata [16, 20] middleware between Django portal and the remote HPCs. Apache Airavata is a open source middleware platform that is built to communicate with a spectrum of HPCs, from traditional to cloud resources, both national and international. The Apache Airavata middleware provides gateway services such as user authorization and authentication, managing user data storage, configuring LROSE tools, and data sharing capabilities for gateway users. All these services are accessible through the Django portal, which is extended to incorporate the workflows.

Four areas of software development connect the flow of data from observations to final rainfall estimations: 1) a default Wagtail Content Management System (Wagtail CMS) [1] to implement gateway theme and documentation, 2) shell scripts that run as jobs on the Jetstream virtual cluster [6], 3) the gateway application programming interface (API) that provides connections to query and discover the data products, applications, and services offered by the gateway, and 4) a JavaScript (jQuery, Vue JS) HTML plugin that allows a custom web interface for the workflows [4] in the Django portal. For the Rainfall Estimation workflow, we take advantage of the custom Django application interface. Figure 4 illustrates the architecture of the platform in a flowchart style.

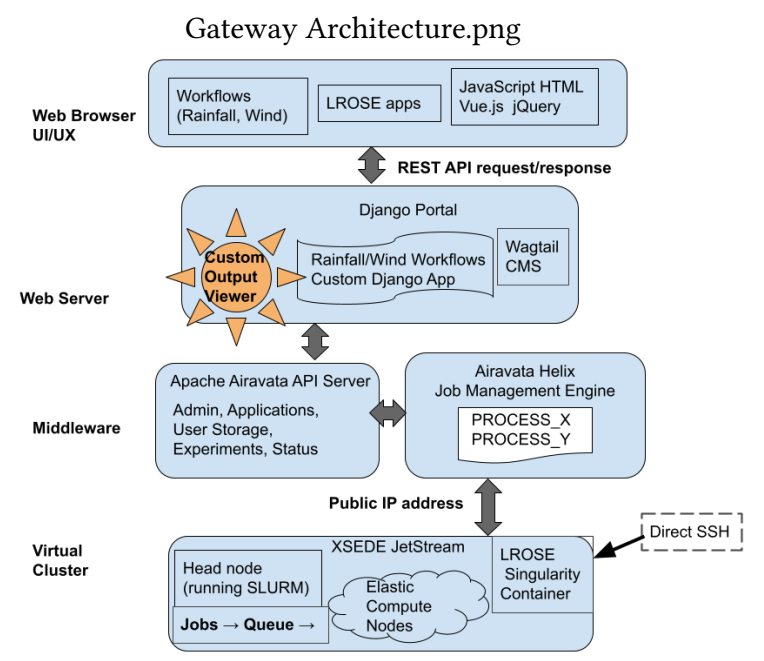

Figure 4: LROSE Gateway Architecture.

2.2.1 Django Portal. The Django portal offers an interactive way to quickly populate a gateway with applications (Figure 5). The existence of user management is a huge boost to the LROSE gateway development. It is easy to add new applications in the Django Settings, to customize inputs and outputs, environment variables, and connect compute resources. The gateway interfaces provide easy access to the Jetstream virtual cluster that connects to our XSEDE [21] compute resource allocation.

Researchers often view results by scanning through images by time, or by radar data field. This requires a custom display of the images with a selector by time or by data field. The Django portal 


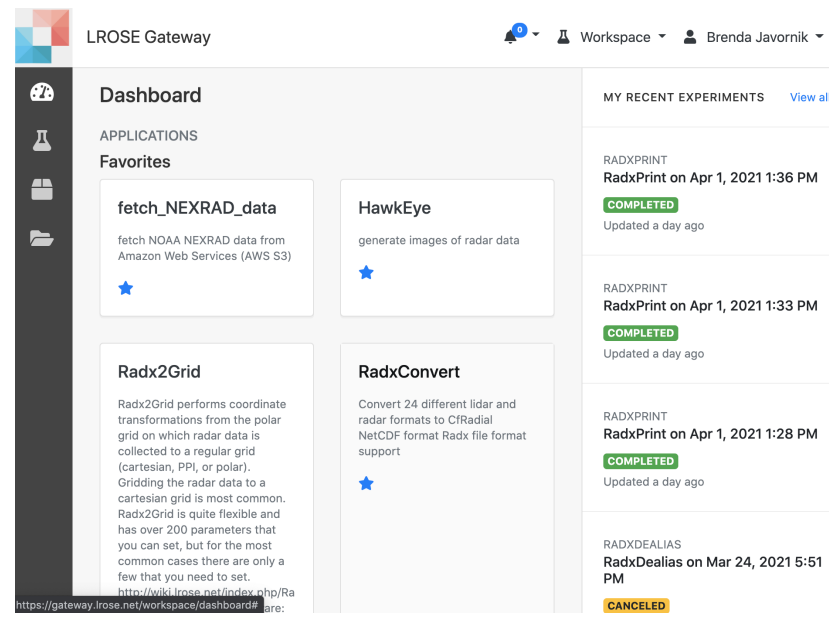

Figure 5: LROSE individual tools implemented as applications in the gateway prototype.

offers a plugin capability to customize input or output to an application. A custom output viewer is a Python class with a specific function named, generate_data. Generate_data returns an image as a byte stream. Within the generate data function we utilize Airavata's SDK to get the user storage which is where the output files are stored. We loop through all of the Data Product URI's to get the specific experiment's file names. Then we look through each file to determine if the specific field is included within the file name, and if it is we then open that file to be displayed. The generate_data function has a return statement and within that return statement we provide the user with options for different image types such as DBZ, PHIDP, RHOHV, etc. When a user selects a certain image type that field is sent to the generate_data function. We parse out all other image types to show only the desired image for the user. This functionality gives the user a more centralized and friendly view of the different image types LROSE provides. Figure 6 is an example of the custom output viewer.

2.2.2 Bash Shell Scripts. The shell scripts use Slurm directives to specify the resource requirements and job parameters [12]. The shell scripts invoke Singularity containers to run a sequence of LROSE tools. [13]. All the LROSE tools run inside Singularity containers.

2.2.3 The Gateway API. An implementation concern was access to data from a previous stage without copying large data files. A typical analysis may use several days of data. The results from one stage need to flow as input into the next stage. To resolve the issue, we take advantage of the information in the gateway API. All input and output files and arguments to an application reside in a uniquely named process directory on the virtual cluster. Each process directory, is mapped to an experiment in the gateway. We make use of this automatic behavior and query the gateway API for the information.

2.2.4 JavaScript and HTML Plugin. Inside a custom Django application we use JavaScript (with help from jQuery and Vue) to query the gateway API for the name and location of the results from a

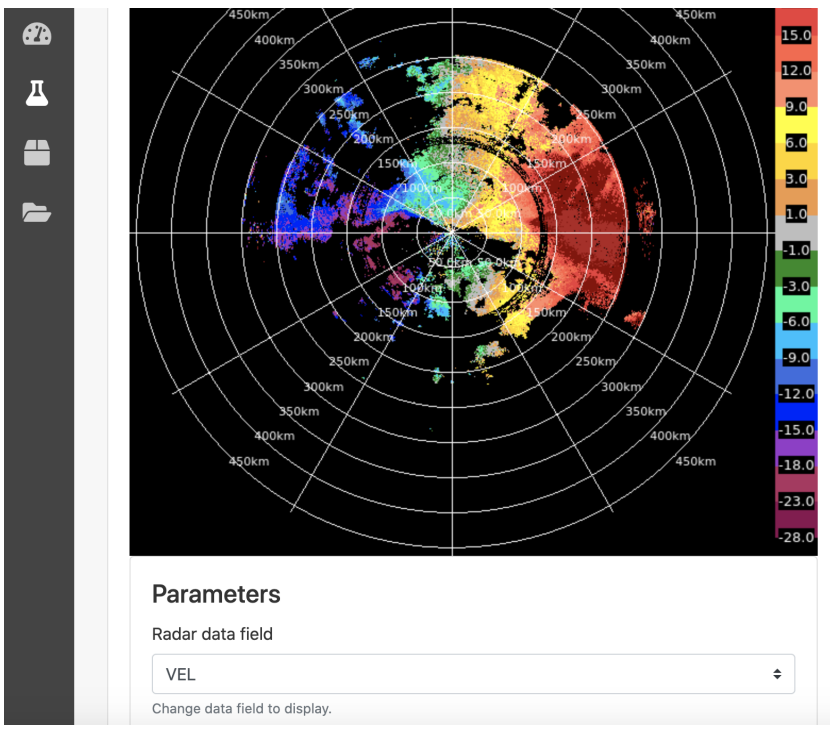

Figure 6: The custom display of velocity after conversion to CfRadial format. The image changes according to the radar data field selected from the drop-down list.

prior stage. The location information is a directory on the virtual cluster.

\subsection{Integration}

Each stage of a workflow becomes an application in the gateway. An experiment captures the inputs, outputs, and shell script, then archives the information as files to a uniquely named, internal directory. The gateway API, provides the name of the archive directory and we pass the name as input to the next stage. The archive directory arrives as an argument to a shell script. The shell script uses the bind option to connect the archive directory to a local file system inside the Singularity container. This mechanism connects data from one stage of a workflow to the next stage, without copying data files. Every run of a workflow stage creates a new, unique archive directory that records the information needed to reproduce the results. Figure 7 is a screenshot of the second stage of the workflow.

\subsection{Deployment}

The SciGaP group provisioned and deployed the initial science gateway for LROSE (gateway.lrose.net). For the custom Django application that implements the workflows, we maintain a separate Github repository from which the Apache Airavata group can pull updates and integrate the changes with the deployed LROSE gateway. The LROSE Gateway API is immediately available to anyone who has authorization and wants to directly access the applications, and workflow stages. For example, LROSE is a web service for other websites, applications, and the rest of the LROSE community interests. 


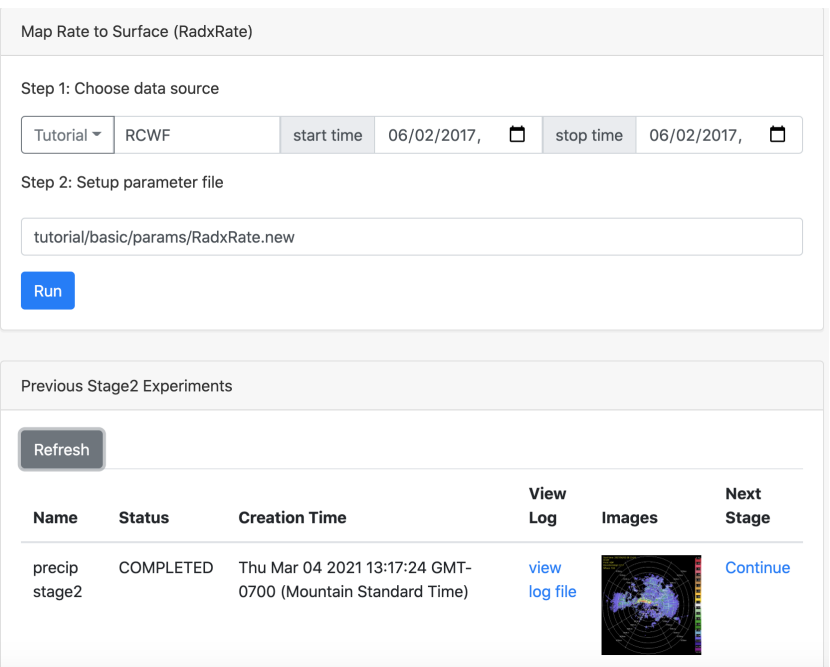

Figure 7: The Rainfall Estimation Workflow. Each completed run of a particular stage of the analysis has a Continue option. The Continue option automatically moves to the next stage and populates the input with a suggested parameter file and data files from the previous stage.

\section{DISCUSSION AND CONCLUSION}

Each stage of a workflow uses at least one LROSE tool, a unique parameter file, and a data set, most often indexed by date and time. Each stage produces images to inspect the results. Tweaks to a parameter file customize the LROSE tool.

Something that is lurking in the background is metadata and it is worthwhile to clarify the management of metadata. Metadata about the radar data is held in the radar files themselves. There is the space extent of the data, the location, and how the beam bends. There is the time side of things: start and end times of a radar data volume, and each ray has time associated with it. The LROSE tools RadxPrint and PrintMdv extract this information from the file headers. Metadata about the experiments and workflows include command-line arguments and parameter files to the LROSE tools during an analysis. Time is the major concern (the start and end time of the analysis) along with radar names. Both an ASCII file and the Slurm job script capture the runtime parameters set in the GUI interactions. The unique PROCESS directory keeps the job script and the ASCII file. Parameter files contain most of the metadata for an individual LROSE tool, or a stage in a workflow. Parameter files capture the complexity of a tool and control the analysis. Each LROSE tool generates its closely tied parameter file. Each parameter is self-documenting in that a programmer must describe the parameter in a paramdef file when adding it to the LROSE tool.

The custom Django application for the workflows is an extra amount of work beyond the available content management system. One wonders if the extra effort is worth it. The custom workflows embed domain expert knowledge about the LROSE tools for a particular type of analysis. Although the tools work in most any order, often dependencies dictate a sequence for a particular result. The custom workflows guide a novice through an analysis. The customized workflows hide the details of argument selection, navigation back and forth between experiment results to find intermediate data, and parameter tuning. The extra work of automating a consistent workflow makes the analysis easier to learn and provides a starting example for further customization and embellishment. A science gateway provides access to the LROSE tools and data with an interface that is simple and hopefully, obvious to use.

\section{FUTURE WORK}

Work continues with the goal of the LROSE Science Gateway to offer applications as a service. Five to ten early adopters will soon test the gateway and precipitation workflow. The workflows and individual applications need to operate at scale on realistic size data sets. The LROSE theme, style sheets, and icons need integration into the LROSE Science Gateway as designed for the UX/UI. Two wind analysis workflows need addition to the gateway. Future LROSE workshops plan to use the gateway for scientific tutorials. Eventually, instructors and researchers may be able to create, share and publish their own custom workflows. The ability to share, publish, and reproduce experimental results would help support the weather radar community.

\section{SOFTWARE AVAILABILITY}

The initial prototype LROSE Science Gateway is available at gateway. lrose.net. The LROSE main website is lrose.net. The source code for LROSE is available from github/NCAR/lrose-core and github/ mmbell/vortrac, github/mmbell/fractl, and github/mmbell/samurai.

\section{ACKNOWLEDGMENTS}

Special thanks to the amazing and wonderful people at SGCI, the Apache Airavata group (Eroma Abeysinghe, Suresh Marru, Sudhakar Pamidighantam, Marcus Christie, Eric Coulter) that assisted in the research, building, and preparation of this work and to the UX/UI group (Yu-Chuan (Chrissy) Chen, Kaitlin Ayako Groothuis, and Paul Parsons) for bringing our ideas into a cohesive, and clear user interface and experience.

LROSE is based on collaborative open-source development, supported by a 4-year SI2-SSI grant number 1550597 from NSF to CSU and NCAR/EOL.

This work uses the Extreme Science and Engineering Discovery Environment (XSEDE) - supported by NSF grant number ACI1548562, this work uses XSEDE Jetstream at IU/TACC through allocation EES200002.

\section{REFERENCES}

[1] Stephen Paul Adithela, Marcus Christie, Suresh Marru, and Marlon Pierce. 2018. Django Content Management System Evaluation and Integration with Apache Airavata. In Practice and Experience in Advanced Research Computing (PEARC '18). ACM, New York, NY, USA. https://doi.org/10.1145/3219104.3229272

[2] Amazon Web Services Public Sector Blog Team. [n.d.]. New Tools for Using Real-Time and Archived NEXRAD Weather Data on AWS. Retrieved January 6 , 2016 from https://aws.amazon.com/blogs/publicsector/nexrad/

[3] Michael M. Bell and Bruno Melli. 2020. nsf-lrose/lrose-cyclone: lrose-cyclone release 20200110. Colorado State University and National Center for Atmospheric Research. https://doi.org/10.5281/zenodo.3604387

[4] Marcus Christie, Suresh Marru, Eroma Abeysinghe, Dimuthu Upeksha, Sudhakar Pamidighantam, Stephen Paul Adithela, Eldho Mathulla, Aarushi Bisht, Shivam Rastogi, and Marlon Pierce. 2020. An extensible Django-based web portal for 
Apache Airavata. In Practice and Experience in Advanced Research Computing (PEARC '20). ACM, New York, NY, USA. https://doi.org/10.1145/3311790.3396650

[5] Marcus Christie, Suresh Marru, Eroma Abeysinghe, Dimuthu Upeksha, Sudhakar Pamidighantam, Stephen Paul Adithela, Eldho Mathulla, Aarushi Bisht, Shivam Rastogi, and Marlon Pierce. 2020. An extensible Django-based web portal for Apache Airavata. In Practice and Experience in Advanced Research Computing 160-167.

[6] J. Eric Coulter, Eroma Abeysinghe, Sudhakar Pamidighantam, and Marlon Pierce. 2019. Virtual Clusters in the Jetstream Cloud: A story of elasticized HPC. In Humans in the Loop: Enabling and Facilitating Research on Cloud Computing (HARC '19). ACM, New York, NY, USA. https://doi.org/10.1145/3355738.3355752

[7] M. Dixon, W.-C. Lee, B. Rilling, , and C. Burghart. [n.d.]. CfRadial Data File Format: Proposed CF-compliant netCDF Format for Moments Data for RADAR and LIDAR in Radial Coordinates. www.eol.ucar.edu/system/files/CfRadialDoc.v1.3. 20130701.pdf

[8] Stephen Lien Harrell. 2016. WeatherPipe. Texas Advanced Computing Center TACC, Austin, Texas. https://github.com/stephenlienharrell/WeatherPipe

[9] M. Heistermann, S. Collis, M. J. Dixon, S. Giangrande, J. J. Helmus, B. Kelley, J. Koistinen, D. B. Michelson, M. Peura, T. Pfaff, and D. B. Wolff. 2015. The Emergence of Open-Source Software for the Weather Radar Community. Bull. Amer. Meteor. Soc. 96 (2015), 117-128. https://doi.org/10.1175/BAMS-D-1300240.1

[10] M. Heistermann, S. Collis, M. J. Dixon, J. J. Helmus, A. Henja, D. B. Michelson, and Thomas Pfaff. 2015. An Open Virtual Machine for Cross-Platform Weather Radar Science. Bulletin of the American Meteorological Society 96 (October 2015), 1641-1645. Issue 10. https://doi.org/10.1175/BAMS-D-14-00220.1

[11] J.J. Helmus and S.M. Collis. 2016. The Python ARM Radar Toolkit (Py-ART) a Library for Working with Weather Radar Data in the Python Programming Language. Fournal of Open Research Software 4, 1 (2016), e25. https://doi.org/10. 5334/jors. 119

[12] Morris A. Jette, Andy B. Yoo, and Mark Grondona. 2002. SLURM: Simple Linux Utility for Resource Management. In In Lecture Notes in Computer Science: Proceedings of Job Scheduling Strategies for Parallel Processing (7SSPP) 2003. SpringerVerlag, 44-60

[13] GM Kurtzer, V Sochat, and MW Bauer. 2017. Singularity: Scientific containers for mobility of compute. PLoS ONE 12, 5 (2017). https://doi.org/10.1371/journal. pone. 0177459

[14] Katherine A Lawrence, Michael Zentner, Nancy Wilkins-Diehr, Julie A Wernert, Marlon Pierce, Suresh Marru, and Scott Michael. 2015. Science gateways today and tomorrow: positive perspectives of nearly 5000 members of the research community. Concurrency and Computation: Practice and Experience 27, 16 (2015), $4252-4268$.

[15] Katherine A. Lawrence, Michael Zentner, Nancy Wilkins-Diehr, Julie A. Wernert, Marlon Pierce, Suresh Marru, and Scott Michael. 2015. Science gateways today and tomorrow: positive perspectives of nearly $5000 \mathrm{mem}$ bers of the research community. Concurrency and Computation: Practice and Experience 27, 16 (2015), 4252-4268. https://doi.org/10.1002/cpe.3526 arXiv:https://onlinelibrary.wiley.com/doi/pdf/10.1002/cpe.3526

[16] Suresh Marru, Lahiru Gunathilake, Chathura Herath, Patanachai Tangchaisin, Marlon Pierce, Chris Mattmann, Raminder Singh, Thilina Gunarathne, Eran Chinthaka, Ross Gardler, et al. 2011. Apache airavata: a framework for distributed applications and computational workflows. In Proceedings of the 2011 ACM workshop on Gateway computing environments. 21-28.

[17] National Centers for Environmental Information (NCEI). [n.d.]. NEXRAD. Retrieved April 7, 2021 from https://www.ncdc.noaa.gov/data-access/radar-data/ nexrad

[18] National Centers for Environmental Information (NCEI). [n.d.]. NEXRAD Products. Retrieved April 7, 2021 from https://www.ncdc.noaa.gov/data-access/radar-data/ nexrad-products

[19] National Centers for Environmental Information (NCEI) formerly known as National Climatic Data Center (NCDC). [n.d.]. Radar Data in the NOAA Big Data Project. Retrieved April 7, 2021 from https://www.ncdc.noaa.gov/dataaccess/radar-data/noaa-big-data-project

[20] Marlon Pierce, Suresh Marru, Eroma Abeysinghe, Sudhakar Pamidighantam, Marcus Christie, and Dimuthu Wannipurage. 2018. Supporting science gateways using apache airavata and scigap services. In Proceedings of the Practice and Experience on Advanced Research Computing. 1-4.

[21] J. Towns, T. Cockerill, M. Dahan, I. Foster, K. Gaither, A. Grimshaw, V. Hazlewood, S. Lathrop, D. Lifka, G. D. Peterson, R. Roskies, J. R. Scott, and N. WilkinsDiehr. 2014. XSEDE: Accelerating Scientific Discovery. Computing in Science \& Engineering 16, 5 (Sept.-Oct. 2014), 62-74. https://doi.org/10.1109/MCSE.2014.80

[22] Nancy Wilkins-Diehr, Michael Zentner, Marlon Pierce, Maytal Dahan, Katherine Lawrence, Linda Hayden, and Nayiri Mullinix. 2018. The Science Gateways Community Institute at Two Years. In Proceedings of the Practice and Experience on Advanced Research Computing. ACM, New York, NY, Article 53. https://doi. org/10.1145/3219104.3219142 